\title{
Modulation of Intestinal Gas Dynamics in Healthy Human Volunteers by the 5-HT 4 Receptor Agonist Tegaserod
}

Radoslav Coleski, Chung Owyang, and William L. Hasler, M.D.

Division of Gastroenterology, Department of Internal Medicine, University of Michigan Medical Center, Ann Arbor, Michigan

OBJECTIVES: Bloating in irritable bowel syndrome (IBS) may result from impaired intestinal gas transit and is reduced by the $5-\mathrm{HT}_{4}$ agonist tegaserod. Abnormal serotonergic function underlies many IBS symptoms, but the role of $5-\mathrm{HT}_{4}$ pathways in regulating gas dynamics under healthy conditions is unexplored. We hypothesized that $5-\mathrm{HT}_{4}$ activation by tegaserod stimulates gas transit in healthy individuals.

METHODS: $\quad$ Sixteen normal volunteers underwent jejunal perfusion of gas mixtures $\left(88 \% \mathrm{~N}_{2}, 5.5 \% \mathrm{O}_{2}, 6.5 \% \mathrm{CO}_{2}\right)$ at $11.2 \mathrm{~mL} / \mathrm{min} \times 3 \mathrm{~h}$ under control conditions and $3 \mathrm{~h}$ after oral tegaserod $6 \mathrm{mg}$ on separate days. Gas collected from an intrarectal catheter was quantified using a barostat.

RESULTS: $\quad$ Under control conditions, gas evacuation after a lag period $(1,959 \pm 428 \mathrm{~s})$ was predominantly pulsatile with expulsion of $1,984 \pm 90 \mathrm{~mL}$. A mean of $29 \pm 2$ boluses with volumes of $72 \pm 5 \mathrm{~mL}$ were expelled. In 10 subjects with physiologic degrees of gas retention in control studies $(248 \pm$ $73 \mathrm{~mL}$ ), tegaserod increased expulsion from $1,768 \pm 73$ to $1,973 \pm 37 \mathrm{~mL}$ and decreased retention to $43 \pm 37 \mathrm{~mL}(p<0.05)$. Total volumes expelled as boluses were greater after tegaserod $(1,708 \pm$ 73 vs $1,846 \pm 59 \mathrm{~mL}, p<0.05$ ) from increased bolus numbers in four subjects and increased bolus volumes in seven. Nonpulsatile continuous flow tended to increase with tegaserod (43 \pm 7 vs $126 \pm$ $43 \mathrm{~mL}, p=0.10$ ). Tegaserod did not increase evacuation in individuals without physiologic gas retention.

CONCLUSIONS: The 5- $\mathrm{HT}_{4}$ agonist tegaserod promotes evacuation of jejunally perfused gas mixtures in healthy humans. These findings provide the foundation for future investigations into use of $5-\mathrm{HT}_{4}$ agonists in conditions of pathologic gas retention.

(Am J Gastroenterol 2006;101:1858-1865)

\section{INTRODUCTION}

Bloating is a prevalent complaint in the general population and in patients with irritable bowel syndrome (IBS) (1-3). The pathophysiology of bloating is incompletely understood, although gut motor and sensory components likely participate in symptom generation. Recent studies suggest that IBS patients exhibit abnormal retention of a physiologic gas mixture perfused in the proximal jejunum, suggesting the presence of retarding motor patterns in the small intestine or colon that may contribute to development of gaseous symptoms $(4,5)$. In theory, a pharmaceutical agent that can stimulate intestinal gas flow might have beneficial effects on symptoms resulting from impaired gas transit including bloating.

Serotonergic pathways play important roles in the modulation of gut motor activity. In many species, activation of $5-\mathrm{HT}_{4}$ receptors elicits increased peristalsis in isolated gut segments $(6,7)$. In preliminary studies, we have demonstrated enhancement of the colonic peristaltic reflex in healthy humans with $5-\mathrm{HT}_{4}$ receptor stimulation (8). Conversely, in- hibition of gut transit has been observed with both $5-\mathrm{HT}_{3}$ and $5-\mathrm{HT}_{4}$ receptor antagonism $(9,10)$. The $5-\mathrm{HT}_{4}$ receptor agonist tegaserod has demonstrated potent prokinetic effects on small intestinal and colonic transit in IBS patients with constipation (11). In addition, this drug has been reported to reduce symptoms of bloating in constipation-predominant IBS patients suggesting possible effects on luminal gas flow via unknown mechanisms (12). To better understand the beneficial actions of tegaserod in reducing gaseous symptoms, it is important to define the physiologic importance of $5-\mathrm{HT}_{4}$ receptor modulation of intestinal gas transit under conditions of health.

In the present study, we employed a validated method to test the hypothesis that $5-\mathrm{HT}_{4}$ receptor activation stimulates gas transit in healthy individuals (13). Specifically, a physiologic gas mixture was jejunally perfused in healthy human volunteers under control conditions and after tegaserod on separate days. Several gas dynamic parameters were quantified to gain insight into the prokinetic actions of the drug. Results were compared in individuals with physiologic degrees 
of gas retention under control conditions to those subjects with no retention to assess if the basal rate of gas transit influences responses to the $5-\mathrm{HT}_{4}$ agonist. Through these investigations, we hoped to gain insight into the participation of $5-\mathrm{HT}_{4}$ receptor pathways in the control of intestinal gas dynamics under healthy conditions.

\section{METHODS}

\section{Test Subjects}

Sixteen healthy volunteers ( 12 men, 4 women, mean age $30 \pm$ $3 \mathrm{yr}$, range 19-49 yr) with no history of gastrointestinal symptoms, no prior gastrointestinal surgery, and on no medications known to alter gut motor function or transit were recruited through campus-wide advertisement. Each subject gave written informed consent before entering the study. Women of child-bearing potential underwent serum pregnancy testing prior to study enrollment. All studies were approved by the University of Michigan Health System Institutional Review Board.

\section{Study Design}

All subjects underwent two intestinal gas dynamic studies on separate days at least 7 days apart. Studies were performed under control conditions in which subjects received no treatment and $3 \mathrm{~h}$ after oral administration of $6 \mathrm{mg}$ of tegaserod (Novartis Pharmaceuticals, East Hanover, NJ) in a crossover fashion in random order. It was necessary to begin the gas studies $3 \mathrm{~h}$ after drug administration because tegaserod frequently elicited a diarrheal response in the first 60-90 min which occluded the rectal collection catheter in preliminary validation studies. As tegaserod exhibits an elimination halflife of 5.8-9.8 h, significant drug effects were expected to persist beyond the end of the jejunal gas perfusion period (14). Volunteers fasted overnight for at least $8 \mathrm{~h}$ and reported at 7:30 a.m. on each study day. Subjects underwent initial rectal evacuation with a $500 \mathrm{~mL}$ water enema. A silicone rubber catheter with an outside diameter of $4.2 \mathrm{~mm}$ and a $1.9 \times$ $2.4 \mathrm{~mm}$ central lumen open at its tip (Adult APD Assembly, Dentsleeve Pty Ltd, Wayville, SA, Australia) was passed using fluoroscopy without sedation with positioning of the tip $5 \mathrm{~cm}$ distal to the ligament of Treitz for gas perfusion. A 24-Fr Foley catheter was inserted into the rectum for gas collection. The subject was placed in a quiet, isolated room and lay supine at a $30^{\circ}$ incline for a $60 \mathrm{~min}$ equilibration period prior to initiation of jejunal gas perfusion.

\section{Jejunal Gas Perfusion}

A gas mixture containing $88 \% \mathrm{~N}_{2}, 5.5 \% \mathrm{O}_{2}$, and $6.5 \% \mathrm{CO}_{2}$ was perfused into the jejunum for $3 \mathrm{~h}$ at a rate of $11.2 \mathrm{~mL} / \mathrm{min}$ through the central channel of the duodenojejunal catheter as described and validated previously (13). This mixture of gases approximates the concentrations in venous blood and thus has been termed physiologic (15). Furthermore, using this perfusion protocol with inclusion of a marker gas, there is no significant gas reflux into the stomach and $95 \%$ of perfused gas is recovered in the rectum indicating that unobserved gas losses are minimal. Gas perfusion was controlled by a digital mass flow controller (Brooks ${ }^{\circledast}$ Smart Mass Flow Controller, Model 5850S, Brooks Instrument, Hatfield, PA) and flow entering the jejunum was monitored by a digital mass flow meter (Brooks ${ }^{\circledR}$ Smart Flow Meter, Model 5860E). The mass flow controller and the mass flow meter were interfaced with a personal computer (Dimension 8200, Dell Inc., Round Rock, TX) via a digital converter (USB data Acquisition Function Module, Data Translation Inc, Marlboro, MA). Rates of gas perfusion were set and monitored using Data Acquisition Software Version 1.0 (Data Translation Inc, Marlboro, MA).

\section{Quantification of Evacuated Gas}

Evacuated gas collected from the rectal catheter was collected via leak-proof, low resistance, low-compliance tubing and was measured and recorded in real-time by a barostat (Isobar III, G\&J Electronics, Hamilton, Canada) which was controlled by Protocol Plus ${ }^{\mathrm{TM}}$ Deluxe software (G\&J Electronics). Prior to each study, the barostat was emptied and the pressure was set at $0 \mathrm{mmHg}$ with a sensitivity of $0.3 \mathrm{mmHg}$ and a maximum flow rate of $35 \mathrm{~mL} / \mathrm{s}$. Any gas flow generating a pressure $>0.3 \mathrm{mmHg}$ was collected into the barostat. Volumes and pressures of gas collected in the barostat were recorded over the 3-h perfusion period on the personal computer for subsequent analysis.

\section{Analysis of Intestinal Gas Dynamic Parameters}

After an initial lag period, gas was evacuated from the rectum either as bolus movements or as continuous flow during jejunal gas perfusion. The lag period was defined as the time from initiation of gas perfusion to the first passage of $>20 \mathrm{~mL}$ of gas from the rectal catheter. Bolus movements were defined as passages $>10 \mathrm{~mL}$ in volume at a rate $>2 \mathrm{~mL} / \mathrm{s}$ with an interval between movements $>10 \mathrm{~s}$ regardless of the pressure of the gas movement. The volume, duration, and peak pressure for each bolus movement were determined as shown in Figure 1. The flow rate for each bolus movement was calculated by dividing the bolus volume by its duration. Continuous flow was determined as a gas movement that generated an output $<10 \mathrm{~mL}$ or at a rate $<2 \mathrm{~mL} / \mathrm{s}$. These criteria have previously been validated and published and clearly distinguish two distinct forms of gas evacuation (13). Visual inspection of gas expulsion profiles during bolus evacuations show well-defined evacuations of approximately $70 \mathrm{~mL}$ that last for roughly $5 \mathrm{~s}$. Setting limits of $10 \mathrm{~mL}$ for the volume of the bolus, $2 \mathrm{~mL} / \mathrm{s}$ for bolus flow rates, and $10 \mathrm{~s}$ between boluses provides nearly a $100 \%$ detection of bolus movements. Conversely, visual examination of gas expulsion profiles during periods of continuous flow show discrete periods in which evacuation proceeds in linear rather than pulsatile fashion (i.e., no miniature boluses $<10 \mathrm{~mL}$ are observed), whereas inspection of other parts of the same tracings show no gas elimination, 

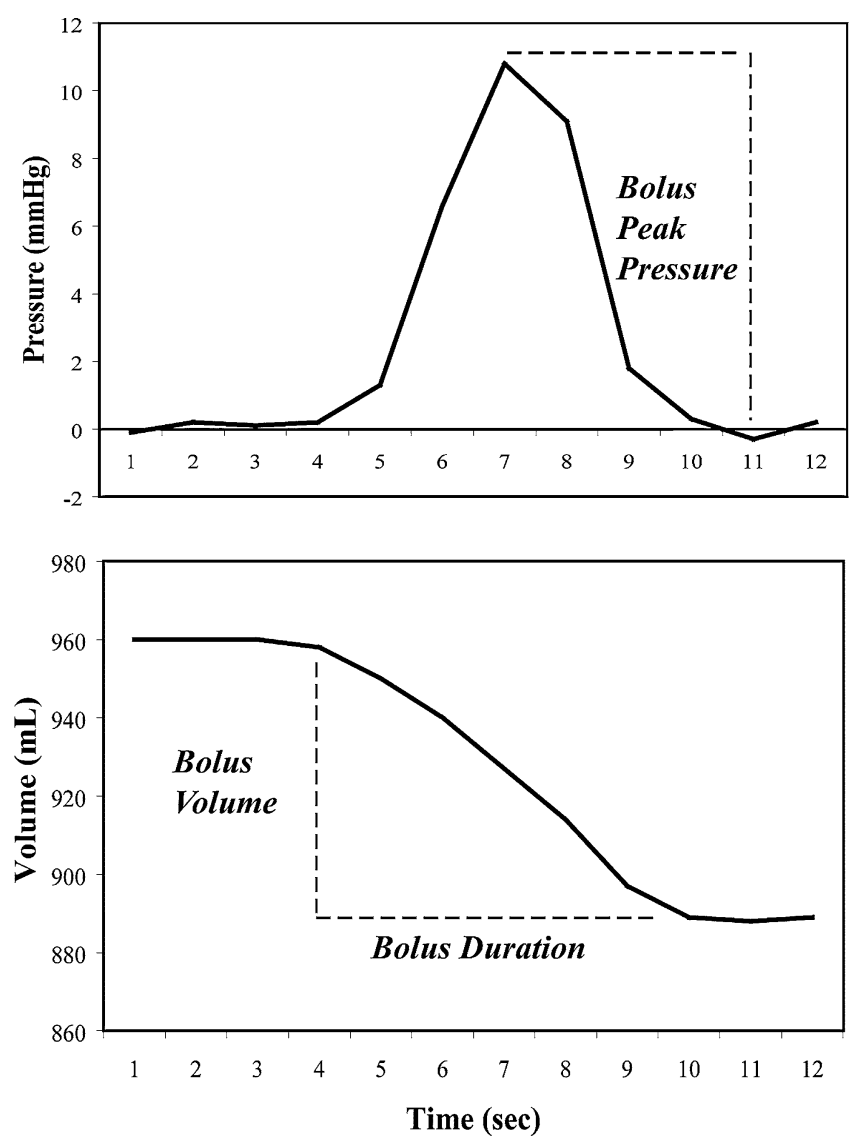

Figure 1. Sample pressure and volume tracings from the barostat are shown during a bolus gas evacuation from the rectum. On the upper tracing, peak bolus pressure is defined as the pressure difference between the maximal pressure and the baseline pressure. On the lower tracing, bolus volume and duration are displayed. The bolus volume is the volume difference between the volume before and after the gas evacuation. The bolus duration is the time needed for $90 \%$ of the bolus volume to be evacuated.

indicating continuous flow does not result from collection system leaks. The total gas volume evacuated during jejunal perfusion at $3 \mathrm{~h}$ was calculated as the sum of bolus and continuous gas flow volumes. The volume of gas retained within the gut at $3 \mathrm{~h}$ was calculated as the difference between the volume of gas perfused and the total volume of gas recovered. One goal of the study prior to initiating experimentation was to determine if an individual's gas transit profile influenced the response to tegaserod. Thus, gas dynamic parameters were compared in volunteers with physiologic degrees of gas retention (defined as $<2,016 \mathrm{~mL}$ expelled at the end of the 3 -h gas perfusion period) and those without retention (defined as $\geq 2,016 \mathrm{~mL}$ expelled at the end of the jejunal perfusion).

\section{Statistical Analysis}

All results are expressed as mean \pm SEM. Intestinal gas dynamic parameters including lag times to first evacuation, bolus volume, bolus pressure, bolus flow rate, and continuous flow rate were compared under control conditions and after tegaserod. Two-tailed Student's testing was performed
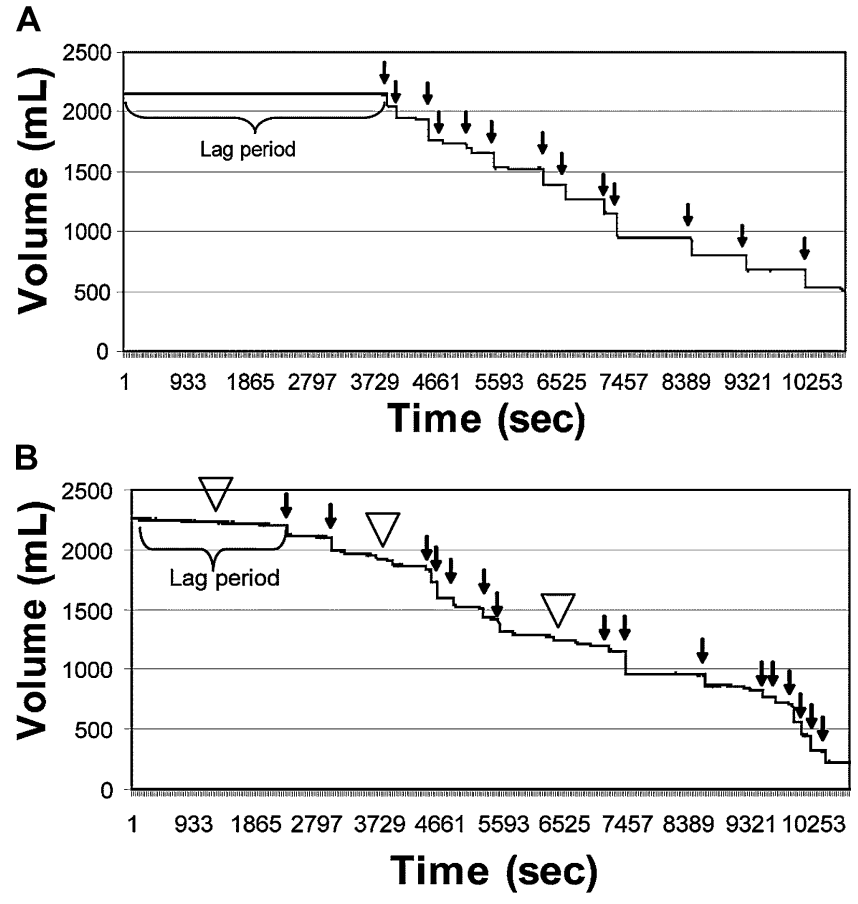

Figure 2. Sample gas evacuation profiles are shown for a healthy individual under control conditions $(A)$ and beginning $3 \mathrm{~h}$ after administration of tegaserod $6 \mathrm{mg}(B)$. In each case, gas evacuation is pulsatile with passage of discrete boluses (arrows) after a lag period after initiation of jejunal gas perfusion during which time no gas expulsion occurs. The tracing after tegaserod is characterized by passage of greater numbers of gas boluses with a larger total volume of gas expelled at $3 \mathrm{~h}$. Open arrowheads signify periods of continuous gas expulsion not in the form of bolus evacuations, seen in this individual primarily after tegaserod.

as appropriate to determine significant differences between gas dynamic parameters. Paired analyses were performed for within-group comparisons, whereas unpaired analyses were performed for comparisons between different subject groups. A $p$-value of $<0.05$ defined statistical significance.

\section{RESULTS}

All volunteers completed both jejunal gas perfusion studies under control conditions and after tegaserod. Representative gas evacuation profiles for one subject are shown in Figure 2. Under control conditions, gas evacuation was predominantly pulsatile in nature and proceeded after a lag period in which no expulsion occurred (Fig. 2A). After tegaserod, most gas evacuation also was bolus in nature although increased periods of continuous gas expulsion not occurring in the form of bolus evacuations were observed (Fig. 2B).

\section{Control Studies}

Of the 16 healthy volunteers, 10 exhibited physiologic degrees of gas retention during control studies and six showed no gas retention during the 3 -h jejunal gas perfusions. Table 1 compares gas dynamic parameters in gas retainers (mean gas 
Table 1. Gas Retention Characteristics of Healthy Volunteers

\begin{tabular}{lcc}
\hline & $\begin{array}{c}\text { Gas Retainers } \\
(\mathrm{N}=10)\end{array}$ & $\begin{array}{c}\text { Nonretainers } \\
(\mathrm{N}=6)\end{array}$ \\
\hline Gas expelled $(\mathrm{mL})$ & $1,768 \pm 73^{*}$ & $2,344 \pm 85$ \\
Gas retained $(\mathrm{mL})$ & $248 \pm 73^{*}$ & $-328 \pm 85$ \\
Lag period $(\mathrm{s})$ & $2,493 \pm 584$ & $1,068 \pm 423$ \\
Bolus number per 3 $\mathrm{h}$ & $26 \pm 3$ & $32 \pm 4$ \\
Mean bolus volume $(\mathrm{mL})$ & $71 \pm 6$ & $75 \pm 7$ \\
Continuous volume $(\mathrm{mL})$ & $44 \pm 7^{\dagger}$ & $68 \pm 9$ \\
\hline
\end{tabular}

${ }^{*} p<0.05$ retainers versus nonretainers.

${ }^{\dagger} p=0.05$ retainers versus nonretainers.

retention $248 \pm 73 \mathrm{~mL}$ ) and nonretainers (mean gas retention $-328 \pm 85 \mathrm{~mL}$ ). Nonretainers showed a trend to shorter lag times to first evacuation that did not reach statistical significance $(p=0.07)$. No differences in numbers of bolus evacuations over $3 \mathrm{~h}(p=0.26)$ and mean bolus volumes $(p=$ $0.70)$ were observed between retainers and nonretainers (Table 1). However, nonretainers exhibited significant increases in amounts of gas expelled continuously not in the form of bolus evacuations ( $p=0.05)$ (Table 1).
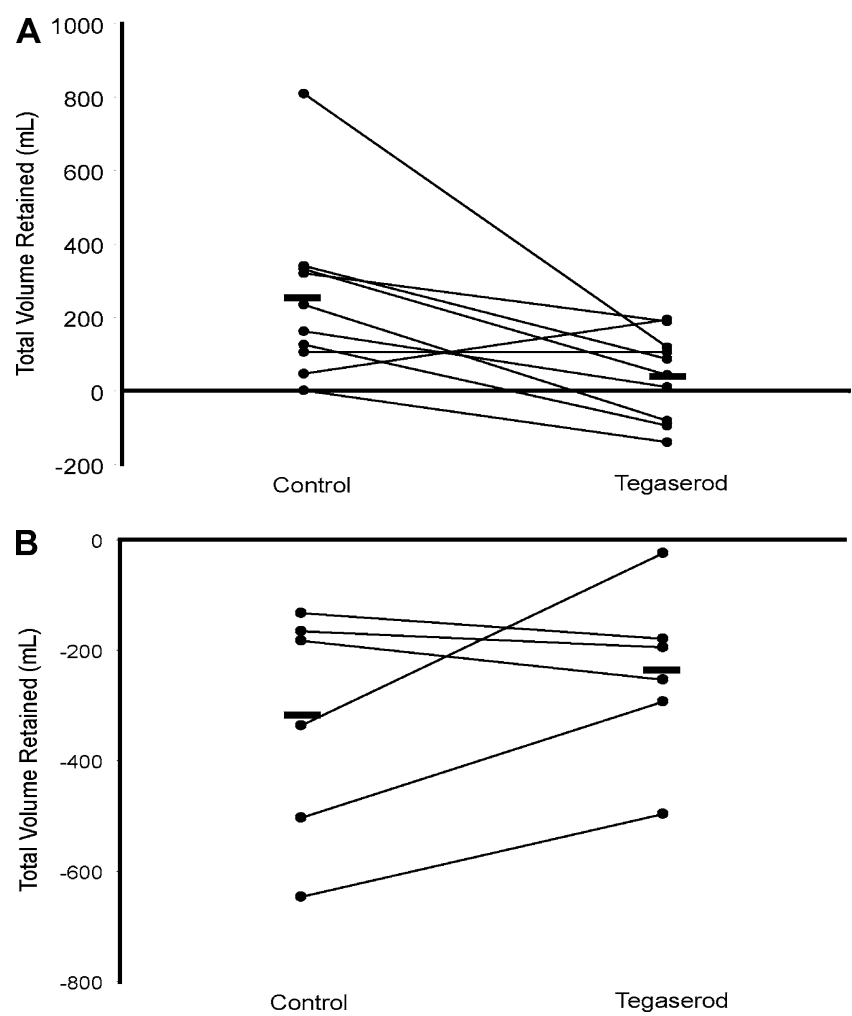

Figure 3. Total volumes of gas expelled during the 3 -h jejunal gas perfusion in control and tegaserod studies are plotted for each volunteer with physiologic degrees of gas retention $(A)$ and without gas retention $(B)$ under control conditions. Horizontal bars represent the mean values for each test condition. The $5-\mathrm{HT}_{4}$ receptor stimulation elicited increased total volumes of gas evacuated in 9 of 10 subjects with gas retention but had no consistent effect in those subjects without basal gas retention.
Table 2. Tegaserod Effects on Gas Bolus Parameters

\begin{tabular}{lcr}
\hline & Control & Tegaserod \\
\hline Volume expelled $(\mathrm{mL})$ & $1,768 \pm 73^{*}$ & $1,973 \pm 37$ \\
Volume retained $(\mathrm{mL})$ & $248 \pm 73^{*}$ & $43 \pm 37$ \\
Bolus volume expelled (mL) & $1,708 \pm 73^{*}$ & $1,846 \pm 59$ \\
Continuous volume expelled $(\mathrm{mL})$ & $43 \pm 7^{\dagger}$ & $126 \pm 43$ \\
Lag period (s) & $2,493 \pm 584$ & $2,004 \pm 388$ \\
Bolus flow rate $(\mathrm{mL} / \mathrm{s})$ & $13.5 \pm 0.8$ & $15.2 \pm 0.9$ \\
Peak bolus pressure $(\mathrm{mmHg})$ & $3.2 \pm 0.6$ & $3.1 \pm 0.3$ \\
Bolus duration $(\mathrm{s})$ & $5.2 \pm 0.2$ & $5.2 \pm 0.3$
\end{tabular}

${ }^{*} p<0.05$ control versus tegaserod.

${ }^{\dagger} p=0.10$ control versus tegaserod

\section{Tegaserod Effects in Individuals with Physiologic Gas Retention}

Tegaserod promoted net gas expulsion in 9 of 10 volunteers with physiologic gas retention in control studies (Fig. 3A). Total volumes of gas expelled during the 3 -h period of gas perfusion were significantly greater after tegaserod $(1,973 \pm$ $37 \mathrm{~mL})$ compared with the control studies $(1,768 \pm 73 \mathrm{~mL}$, $p<0.05$ ) (Table 2). This correlated with a decrease in gas retention on tegaserod (43 $\pm 37 \mathrm{~mL}$ ) compared with control $(248 \pm 73 \mathrm{~mL}, p<0.05)$ (Table 2$)$. In both control and tegaserod studies, gas expulsion was predominantly pulsatile in nature. The volume of gas evacuated as bolus expulsions was significantly enhanced by tegaserod $(1,846 \pm 59 \mathrm{~mL})$ compared with control studies $(1,708 \pm 73 \mathrm{~mL})(p<0.05)$ (Table 2). Taken as a group, tegaserod did not significantly increase numbers of bolus evacuations $(26 \pm 2$ per $3 \mathrm{~h}$ vs $27 \pm$ 3 per $3 \mathrm{~h}$ for control) or bolus volumes $(74 \pm 5 \mathrm{~mL} v s 70 \pm$ $6 \mathrm{~mL}$ for control). Four of 10 volunteers passed greater numbers of bolus expulsions after tegaserod, whereas 7 of 10 passed boluses with greater mean volumes after tegaserod (Figs. 4A, B). When multiplied, the product of bolus number and bolus volume was greater in 9 of 10 volunteers with gas retention under control conditions ( $p<0.05$ ) (Fig. 4C). Volumes of gas expelled in continuous fashion not in the form of bolus evacuations showed a trend to increase with tegaserod $(126 \pm 43 \mathrm{~mL})$ compared with control $(43 \pm 7)$ that did not reach statistical significance $(p=0.10)$ (Table 2$)$. There was no significant difference in lag times from initiation of gas perfusion to the first gas evacuation under control conditions and after tegaserod ( $p=0.46$ ) (Table 2$)$. Other gas dynamic parameters including bolus gas flow rates, peak bolus pressures, and bolus durations were unaffected by tegaserod administration (all $p=$ NS) (Table 2).

\section{Tegaserod Effects in Individuals Without Gas Retention}

Six volunteers did not retain gas during jejunal perfusion of the mixed gas in control studies. In these individuals, total gas volumes expelled over the 3 -h period under control conditions exceeded the volumes perfused (2,016 mL) (Fig. 3B). In the nonretainers, tegaserod had no appreciable effect on lag period to first gas passage $(1,397 \pm 392 \mathrm{~s}$ with tegaserod vs $1,068 \pm 423 \mathrm{~s}$ in controls, $p=0.57$ ) or gas retained 

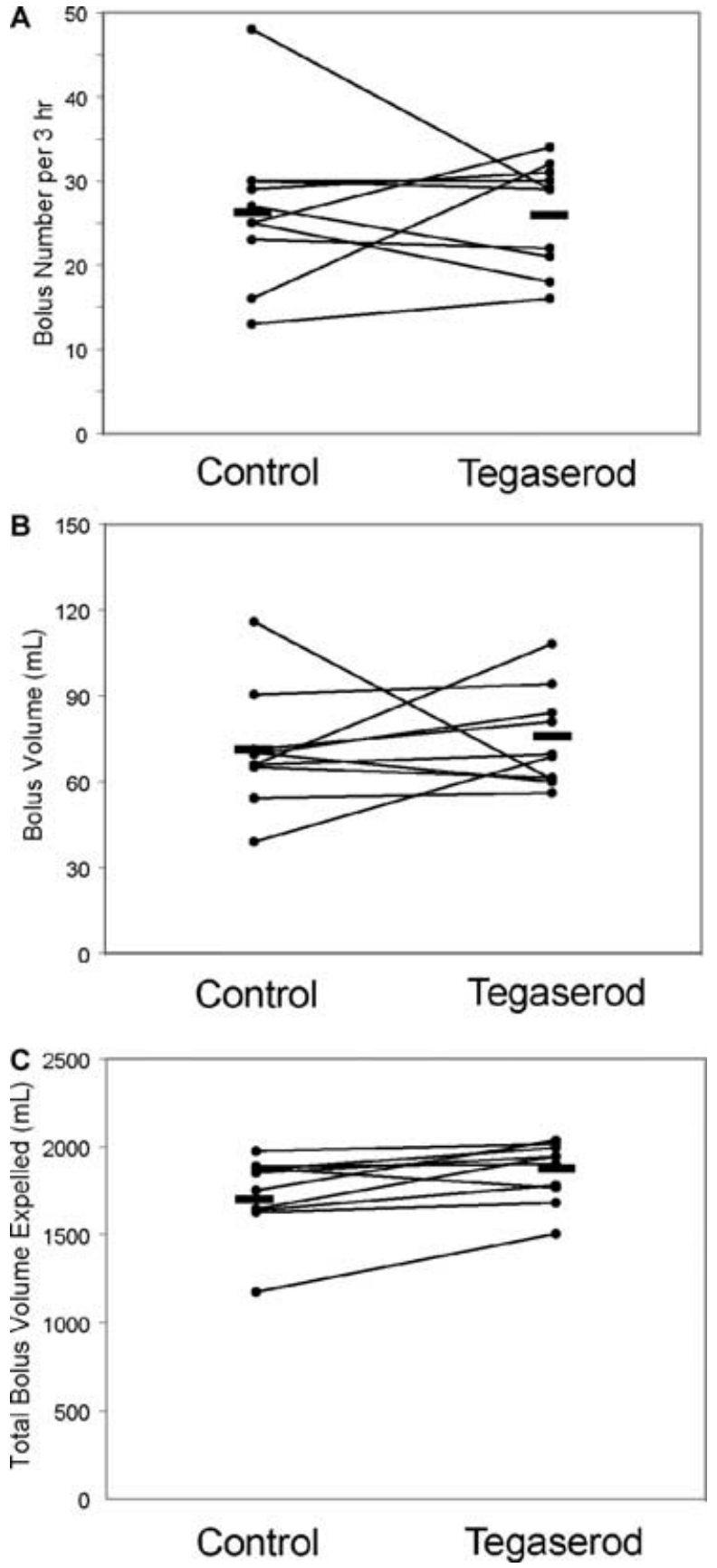

Figure 4. Numbers of bolus evacuations and mean bolus volumes in control and tegaserod studies are plotted for subjects with physiologic degrees of gas retention in control studies. Horizontal bars represent the mean values for each test condition. Four of $10 \mathrm{sub}-$ jects exhibited increased numbers of evacuations $(A)$ whereas 7 of 10 exhibited increased mean bolus volumes $(B)$. When multiplied, the product of bolus number and bolus volume was greater in 9 of 10 volunteers with physiologic gas retention under control conditions (C) $(p<0.05)$.

$(p=0.23)$ (Fig. 3B). Three of six volunteers without gas retention under control conditions exhibited greater gas expulsion and three exhibited less gas expulsion. No individual without retention under control conditions exhibited physiologic gas retention after tegaserod.

\section{DISCUSSION}

Gaseous symptoms including eructation, bloating, and flatulence are prevalent complaints of patients with functional bowel disorders. In an investigation of IBS patients, bloating was experienced on $28 \%$ of days compared with $33 \%$ for abdominal discomfort or pain (2). A second study observed that $66 \%$ of IBS patients report gas as a major symptom (3). Furthermore, $60 \%$ of individuals felt that their gaseous manifestations of IBS were more bothersome than other symptoms such as pain and bowel habit disruption. Our understanding of the pathophysiology of gas and bloating is very limited. As a consequence, most available treatments for bloating are empiric in nature, acting to reduce gas production or adsorb luminal gas collections (16-18). The availability of a pharmaceutical agent that stimulates gas transit would be theoretically beneficial in individuals with pathologic gas retention.

Insight is being provided into the pathogenesis of gas and bloating in the functional bowel disorders using in vivo gas perfusion methods. In a study employing an intestinally perfused argon washout technique, no differences in intraluminal gas volumes were seen in healthy volunteers and individuals with excess gas (19). However, half of the patients exhibited abnormal retention of the perfused argon marker and many showed abnormal retrograde enterogastric flow of the gas suggesting the presence of abnormal gas transit in patients with functional symptoms. However, a second investigation observed no differences in manometric parameters in healthy subjects compared with IBS patients jejunally perfused with nitrogen (20). It was concluded that sensations of bloating and gas retention stemmed from heightened perception rather than an underlying motor dysfunction. Most recently, using a method whereby a physiologic gas mixture is perfused into the jejunum at a slow rate, investigators observed significant gas retention in $90 \%$ of IBS patients compared with $20 \%$ of normal controls, which was associated with symptoms of bloating and increased abdominal girth (4). Development of these models affords the capability to research the role of different neurotransmitter pathways in the regulation of intestinal gas dynamics in health and disease.

Serotonin plays an important role in the control of intestinal propulsion. In most animal models, activation of enteric $5-\mathrm{HT}_{4} / 5-\mathrm{HT}_{1 \mathrm{P}}$ receptors enhances peristaltic reflex activity in isolated gut segments $(6,7,21)$. In some species, additional contributions are provided by $5-\mathrm{HT}_{3}$ receptor activation (22). In healthy humans, rapid distention of a colonic balloon elicits an in vivo peristaltic reflex consisting of an orad contractile response and a caudad relaxation followed by a contraction (23, 24). We have previously shown that this reflex is impaired in some patients with constipation, suggesting its participation in propulsive activities of the lower gut (25). More recently, in preliminary studies, we observed enhancement of both the orad and caudad components of the in vivo colonic peristaltic reflex with $5-\mathrm{HT}_{4}$ receptor activation in healthy humans (8). In clinical studies involving whole gut scintigraphy, 5- $\mathrm{HT}_{4}$ receptor stimulation promotes transit in the stomach, small 
intestine, and colon $(11,26)$. These investigations confirm an important propulsive function for serotonin $\left(5-\mathrm{HT}_{4}\right)$ pathways for luminal solids and liquids. However, there has been no previous study of serotonergic regulation of intestinal gas transit.

In the present study, we employed validated methods to investigate the effects of $5-\mathrm{HT}_{4}$ receptor stimulation on intestinal gas dynamics in healthy human volunteers. Specifically, we demonstrated significant propulsive effects of $5-\mathrm{HT}_{4}$ receptor stimulation on transit of a jejunally perfused gas mixture in healthy humans with physiologic degrees of gas retention under control conditions using the selective $5-\mathrm{HT}_{4}$ receptor agonist tegaserod. In a previous study, the acetylcholinesterase inhibitor neostigmine was shown to produce gas evacuation in patients with functional bloating (27). In contrast, tegaserod had little effect in individuals without gas retention during jejunal gas perfusion. As most healthy individuals have only $150-200 \mathrm{~mL}$ of luminal gas prior to study and as this subject subgroup evacuated $>200 \mathrm{~mL}$ excess gas than was perfused, it is unlikely that any clear-cut gas transit acceleration would be observable in these volunteers (19). Indeed, two individuals expelled $>400 \mathrm{~mL}$ gas than was perfused under control conditions. This observation suggests that some individuals present for study with greater than average amounts of intrinsic gas retention, the evacuation of which must have been facilitated by the gas perfusion methods employed in this study.

The mechanisms by which $5-\mathrm{HT}_{4}$ receptor activation promotes gas flow are uncertain; however, several observations from the present investigation provide insight into the motor stimulatory actions of tegaserod. The main effect noted was an increase in bolus evacuations from the rectal catheter. We defined a bolus passage as one greater than $20 \mathrm{~mL}$ in volume occurring at a flow rate $>2 \mathrm{~mL} / \mathrm{s}$. It is conceivable that this threshold may have excluded smaller boluses; however, most boluses observed in our recordings were significantly greater in volume. We have previously speculated that bolus gas evacuations are a consequence of intermittent luminally occlusive motor patterns that control gas transport in the lower gut (13). It is unlikely that this pattern is secondary to retained fecal material because similar bolus evacuation profiles are seen after colonic lavage (28). In preliminary studies, the degree of small intestinal motor activity correlates with both the number and volume of bolus gas evacuations during jejunal gas perfusion (29). Other potential regions which might contribute to bolus transit profiles include the ileocecal junction and colon. To date, we have not examined manometric findings at these anatomic sites in relation to gas passage. We also observed a strong trend to increased continuous gas evacuation not in the form of bolus expulsions. In our experience, this pattern of gas evacuation typically comprises only a small percentage of total gas flow (13). Visual analysis of the volume expulsion profiles during the continuous flow periods showed no obvious pulsatile character and analysis of the pressure profiles showed no increases in rectal pressure. These observations suggest that continuous flow represents a distinct mechanism for gas evacuation in addition to the more prominent bolus expulsions. The motor phenomena responsible for increased continuous flow are uncertain, but may relate to changes in colon wall tone or, less likely, to creation of transient common cavities during the jejunal gas perfusion experiments. The final observation that tegaserod had no effect on the lag time to first gas evacuation suggests that there is little gas within the distal colon in most individuals at the start of each study that can be evacuated by prokinetic stimulation. The lag time likely reflects the need to accumulate gas within the small intestine before it can be transported to the colon and expelled from the rectum. The findings of this study suggest that this parameter may be relatively unaffected by $5-\mathrm{HT}_{4}$ receptor pathways.

Several issues pertain to this investigation. The current study examined gas transit after only a single dose of the $5-\mathrm{HT}_{4}$ receptor agonist. Tegaserod often elicits transient diarrhea which resolves within several days, suggestive of partial desensitization of the motor response to $5-\mathrm{HT}_{4}$ receptor agonism with time $(12,30)$. Further studies will examine if chronic tegaserod administration elicits similar stimulatory effects on gas dynamics. Secondly, it could be questioned whether the water consumed with the active drug might have contributed to the enhanced gas flow observed with tegaserod treatment. However, although no placebo was administered in this investigation, we have previously shown that acute consumption of $240 \mathrm{~mL}$ of water has no effect on any bolus gas expulsion parameter during jejunal gas perfusion (31). Thus, we believe the findings of this study relate to true pharmacologic effects of the administered prokinetic drug. A third theoretical concern is that the enhanced gas flow after tegaserod relates to clearing the colon of potentially obstructing fecal debris, rather than a true effect on gas transit itself. However, we believe this is unlikely as we have previously observed that colonic lavage with polyethylene glycol retards rather than accelerates gas dynamics (28).

Finally although clear-cut acceleration of gas transit was observed after tegaserod in individuals with physiologic degrees of gas retention, these observations cannot be automatically extended to disease states with pathogenic levels of retention. Indeed, in other investigations, IBS patients exhibit significantly greater gas retention (in most cases $>400 \mathrm{~mL}$ ) than the healthy volunteers in our study suggesting that the volumes of retention in our subjects fall within a normal physiologic range (4). Furthermore, in our investigation, the healthy subjects reported minimal bloating during jejunal gas perfusion. This contrasts with prominent bloating symptoms during gas perfusion in IBS patients, suggesting an additional pathogenic defect of visceral sensation in these individuals $(20,4)$. Investigations into the effects of tegaserod on gas transit and perception of gas perfusion would clarify the mechanisms by which this drug reduces gaseous symptoms in patients with constipation-predominant IBS (12).

In conclusion, $5-\mathrm{HT}_{4}$ receptor stimulation promotes evacuation of jejunally perfused gas mixtures in healthy humans who exhibit physiologic degrees of basal gas retention. This 
finding is consistent with the known prokinetic actions of $5-\mathrm{HT}_{4}$ receptor pathways on luminal transport of solids and liquids and provides a foundation for studies of the motor stimulatory effects of tegaserod on gas flow in patients with functional bowel disorders.

\section{ACKNOWLEDGMENT}

This research was funded by a grant 1 K24 DK02726-01 from the National Institutes of Health.

\section{STUDY HIGHLIGHTS}

\section{What Is Current Knowledge}

- The 5- $\mathrm{HT}_{4}$ receptor agonist tegaserod reduces bloating in some patients with irritable bowel syndrome however its physiologic effects on gas transit under healthy conditions are unexplored.

\section{What Is New Here}

- Compared to control conditions, tegaserod stimulated gas expulsion in healthy individuals with physiologic degrees of gas retention that resulted primarily from increases in pulsatile gas evacuation.

- Tegaserod did not increase evacuation in individuals without physiologic gas retention.

- These findings provide the foundation for future investigations into use of 5- $\mathrm{HT}_{4}$ agonists such as tegaserod in conditions of pathologic gas retention.

Reprint requests and correspondence: William L. Hasler, M.D., 3912 Taubman Center, Box 0362 Ann Arbor, MI 48109.

Received December 28, 2005; accepted March 21, 2006.

\section{REFERENCES}

1. Sandler RS, Stewart WF, Liberman JN, et al. Abdominal pain, bloating, and diarrhea in the United States: Prevalence and impact. Dig Dis Sci 2000;45:1166-71.

2. Hahn B, Watson M, Yan S, et al. Irritable bowel syndrome symptom patterns: Frequency, duration, and severity. Dig Dis Sci 1998;43:2715-8.

3. Lembo T, Naliboff B, Munakata J, et al. Symptoms and visceral perception in patients with pain-predominant irritable bowel syndrome. Am J Gastroenterol 1999;94:1320-6.

4. Serra J, Azpiroz F, Malagelada JR. Impaired transit and tolerance of intestinal gas in the irritable bowel syndrome. Gut 2001;48:14-9.

5. Serra J, Salvioli B, Azpiroz F, et al. Lipid-induced intestinal gas retention in irritable bowel syndrome. Gastroenterology 2002; 123:700-6.

6. Costall B, Naylor RJ, Tuladhar BR. 5-HT 4 receptor mediated facilitation of the emptying phase of the peristaltic reflex in the guinea-pig isolated ileum. Br J Pharmacol 1993;110:1572-8.

7. Grider JR, Kuemmerle JF, Jin JG. 5-HT released by mucosal stimuli initiates peristalsis by activating $5-\mathrm{HT}_{4} /$
5- $\mathrm{HT}_{1 \mathrm{P}}$ receptors on sensory CGRP neurons. Am J Physiol 1996;270:G778-82.

8. Sherif A, Coleski R, Sun WM, et al. 5- $\mathrm{HT}_{4}$ receptor stimulation of colonic peristaltic reflex and basal tonic colon activity by tegaserod in healthy humans: Possible mechanisms of its action in constipation (abstract). Gastroenterology 2004;126:109.

9. Pascual D, Alsasua A, Goicoechea C, et al. The involvement of $5-\mathrm{HT}_{3}$ and $5-\mathrm{HT}_{4}$ receptors in two models of gastrointestinal transit in mice. Neurosci Lett 2002;326:163-6.

10. Houghton LA, Jackson NA, Whorwell PJ, et al. 5-HT 4 receptor antagonism in irritable bowel syndrome: Effect of SB-207266-A on rectal sensitivity and small bowel transit. Aliment Pharmacol Ther 1999;13:1437-44.

11. Prather CM, Camilleri M, Zinsmeister AR, et al. Tegaserod accelerates orocecal transit in patients with constipationpredominant irritable bowel syndrome. Gastroenterology 2000;118:463-8.

12. Muller-Lissner SA, Fumagalli I, Bardhan KD, et al. Tegaserod, a 5- $\mathrm{HT}_{4}$ receptor partial agonist, relieves symptoms in irritable bowel syndrome patients with abdominal pain, bloating and constipation. Aliment Pharmacol Ther 2001; 15:1655-66.

13. Gonlachanvit S, Coleski R, Owyang C, et al. Inhibitory actions of a high fibre diet on intestinal gas transit in healthy volunteers. Gut 2004;53:1577-82.

14. Appel-Dingemanse S, Hirschberg Y, Osborne S, et al. Multiple-dose pharmacokinetics confirm no accumulation and dose proportionality of the novel promotile drug tegaserod (HTF 919). Eur J Clin Pharmacol 2001;56:889-91.

15. Serra J, Azpiroz F, Malagelada JR. Intestinal gas dynamics and tolerance in humans. Gastroenterology 1998;115:54250 .

16. Levitt MD. Follow-up of a flatulent patient. Dig Dis Sci 1979;24:652-4.

17. Ramirez FC, Lee K, Graham DY. All lactase preparations are not the same: Results of a prospective, randomized, placebocontrolled trial. Am J Gastroenterol 1994;89:566-70.

18. Jain NK, Patel VP, Pitchumoni CS. Efficacy of activated charcoal in reducing intestinal gas: A double-blind clinical trial. Am J Gastroenterol 1986;81:532-5.

19. Lasser RB, Bond JH, Levitt MD. The role of intestinal gas in functional abdominal pain. N Engl J Med 1975;293:524-6.

20. Galati JS, McKee DP, Quigley EM. Response to intraluminal gas in irritable bowel syndrome. Motility versus perception. Dig Dis Sci 1995;40:1381-7.

21. Grider JR, Foxx-Orenstein AE, Jin JG. 5-Hydroxy-

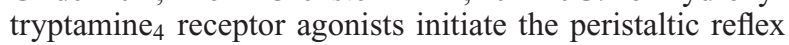
in human, rat, and guinea pig intestine. Gastroenterology 1998; 115:370-80.

22. Tuladhar BR, Costall B, Naylor RJ. 5-HT 3 and 5-HT4 receptor-mediated facilitation of the emptying phase of the peristaltic reflex in the marmoset isolated ileum. Br J Pharmacol 1996;117:1679-84.

23. Sims MA, Hasler WL, Chey WD, et al. Hyperglycemia inhibits mechanoreceptor-mediated gastrocolonic responses and colonic peristaltic reflexes in healthy humans. Gastroenterology 1995;108:350-9.

24. Bjornsson ES, Chey WD, Ladabaum U, et al. Differential 5$\mathrm{HT}_{3}$ mediation of human gastrocolonic response and colonic peristaltic reflex. Am J Physiol 1998;275:G498-505.

25. Bjornsson ES, Chey WD, Hooper F, et al. Impaired gastrocolonic response and peristaltic reflex in slow-transit constipation: Role of 5- $\mathrm{HT}_{3}$ pathways. Am J Physiol Gastrointest Liver Physiol 2002;283:G400-7.

26. Degen L, Matzinger D, Merz M, et al. Tegaserod, a 5-HT 4 receptor partial agonist, accelerates gastric emptying and 
gastrointestinal transit in healthy male subjects. Aliment Pharmacol Ther 2001;15:1745-51.

27. Caldarella MP, Serra J, Azpiroz F, et al. Prokinetic effects in patients with intestinal gas retention. Gastroenterology 2002; 122:1748-55.

28. Gonlachanvit S, Coleski R, Hasler W. Luminal contents modulate intestinal gas transit in healthy humans: Effects of fiber vs. colonic lavage (abstract). Gastroenterology 2003;124:T1802.

29. Gonlachanvit S, Coleski R, Hasler W. Correlation of gas transit with small bowel motility during fasting and postprandial periods in healthy humans (abstract). Gastroenterology 2004;126:107.
30. Tougas G, Snape WJ, Otten MH, et al. Long-term safety of tegaserod in patients with constipation-predominant irritable bowel syndrome. Aliment Pharmacol Ther 2002;16: 1701-8.

31. Gonlachanvit S, Coleski R, Hasler W. Nutrient modulation of intestinal gas dynamics in healthy humans depends on caloric content and physical properties (abstract). Gastroenterology 2003;124:307.

The authors declared no conflicts of interest. 